\title{
INVERSION OF ADJUNCTION FOR LOCALLY COMPLETE INTERSECTION VARIETIES
}

\author{
LAWRENCE EIN AND MIRCEA MUSTAŢÃ
}

\section{INTRODUCTION}

In this note we build on our previous work with Takehiko Yasuda in [EMY], to prove a precise version of Inversion of Adjunction for varieties which are locally complete intersections. In [EMY] is given a description of certain invariants of singularities, minimal log discrepancies, using the codimension of suitable spaces of arcs. This was enough to give Inversion of Adjunction in the case when the ambient variety is smooth. The main new ingredient in this paper is the study of the equations defining the jets which can be lifted to the space of arcs.

Recall the setting for Inversion of Adjunction. Consider a pair $(X, Y)$, where $X$ is a $\mathbb{Q}$-Gorenstein normal variety, and where $Y$ stands for a formal combination $\sum_{i=1}^{k} q_{i} \cdot Y_{i}$, where $q_{i} \in \mathbb{R}_{+}$and $Y_{i} \subset X$ are proper closed subschemes. Given such a pair and a closed subset $W \subseteq X$, one can get an invariant $\operatorname{mld}(W ; X, Y)$, called minimal log discrepancy. Loosely speaking, this invariant comes from evaluating $K_{X}+Y$ along divisors over $X$, with centers in $W$. We refer to Section 3 for the precise definition. The following is our main result.

Theorem 1.1. Let $X$ be a normal, locally complete intersection variety, and $Y=\sum_{i} q_{i} \cdot Y_{i}$, where $q_{i} \in \mathbb{R}_{+}$and $Y_{i} \subset X$ are proper closed subschemes. If $D \subset X$ is a normal effective Cartier divisor such that $D \nsubseteq \bigcup_{i} Y_{i}$, then for every proper closed subset $W \subset D$, we have

$$
\operatorname{mld}(W ; X, D+Y)=\operatorname{mld}\left(W ; D,\left.Y\right|_{D}\right) .
$$

It is a conjecture due to Kollár and Shokurov that the equality in the above conjecture is true if $X$ is just normal and $\mathbb{Q}$-Gorenstein. Note that the equality " $\leq$ " is easy and is known in general. We refer to $\mathrm{K}+$ for a discussion of this conjecture, related topics, and for the proof of some special cases via vanishing theorems. Our approach will be via spaces of arcs, based on the results from [EMY], where the theorem was proved when $X$ is smooth.

Ambro proposed in $\mathrm{Am}$ a conjecture on the semicontinuity of minimal log discrepancies, extending a previous conjecture of Shokurov. This conjecture was proved on an ambient smooth variety in [EMY]. We apply the above theorem to extend this to the locally complete intersection case.

Theorem 1.2. If $X$ is a normal, locally complete intersection variety, and if $Y=\sum_{i} q_{i} \cdot Y_{i}$ is as above, then the function $x \longrightarrow \operatorname{mld}(x ; X, Y), x \in X$, is lower semicontinuous.

1991 Mathematics Subject Classification. Primary 14B05; Secondary 14E30, 14E15, $14 \mathrm{~B} 10$.

Key words and phrases. Jet schemes, minimal log discrepencies, inversion of adjunction. 
Using Theorem 1.1 and the description of minimal log discrepancies in terms of spaces of arcs, we deduce also the following theorem.

Theorem 1.3. Let $X$ be a normal, locally complete intersection variety. $X$ has log canonical (canonical, terminal) singularities if and only if $X_{m}$ is equidimensional (respectively irreducible, normal) for every $m$.

The case of canonical singularities had been conjectured by Eisenbud and Frenkel and proved in $\mathrm{Mu} 2$; the characterization for log canonical singularities appeared there as a conjecture. The statement on terminal singularities answers a question of Mirel Caibăr. All these characterizations have been proved in EMY] in the case when $X$ is a divisor on a smooth variety.

The main ingredient in the proof of Theorem 1.1 is the characterization of minimal log discrepancies in terms of jet schemes from EMY (see Theorem 3.1 below). In order to apply it, we need to estimate the dimension of the subset of a jet scheme, which consists of jets which can be lifted to the space of arcs. This is taken care of in the next section, where we give a small set of equations for this set. In the last section, we use this to give the proofs of the results stated above.

\section{Equations For Liftable Jets}

For definitions and basic properties of jet schemes, we refer to $\mathrm{Mu} 2$. If $X$ is a scheme of finite type over $\mathbb{C}$, then we denote its $m$ th jet scheme by $X_{m}$, and its space of arcs by $X_{\infty}$. When we consider $X_{m}$ and $X_{\infty}$, we restrict ourselves to the $\mathbb{C}$-valued points. If $\psi_{m}^{X}: X_{\infty} \longrightarrow X_{m}$ is the canonical projection, then $X_{m, \infty}$ stands for the image of $\psi_{m}^{X}$.

We work in the ambient space $\mathbb{A}^{n}$, and we identify $\left(\mathbb{A}^{n}\right)_{m}$ with $\left(\mathbb{C}[t] /\left(t^{m+1}\right)\right)^{n} \simeq$ $\mathbb{A}^{(m+1) n}$. The order of $u=\left(u_{i}\right)_{i} \in\left(\mathbb{A}^{n}\right)_{m}$ along an ideal $I \subseteq \mathbb{C}\left[T_{1}, \ldots, T_{n}\right]$ is the minimum of the orders of $P(u) \in \mathbb{C}[t] /\left(t^{m+1}\right)$, over all $P \in I$. Note that if this number is not larger than $m$, then it is well defined.

Let $D=V\left(F_{1}, \ldots, F_{r}\right) \subset \mathbb{A}^{n}$, where $r \leq n$, and $F_{i} \in \mathbb{C}\left[T_{1}, \ldots, T_{n}\right]$, for all $i$. We will denote by $F$ the $r \times 1$ matrix with entries $F_{i}$. Fix $\mathbf{e} \in \mathbb{N}^{r}$ such that $\mathbf{e}_{r} \geq \ldots \geq \mathbf{e}_{1}$, and fix also $p \geq \mathbf{e}_{r}$.

Up to a reordering of the variables, it is enough to consider the following situation. Let $\left(\mathbb{A}^{n}\right)_{p}^{(\mathbf{e})}$ be the set of jets in $\left(\mathbb{A}^{n}\right)_{p}$ satisfying the following conditions. The order along the ideal of $r$-minors of $\left(\partial F_{k} / \partial T_{l}\right)_{k \leq r, l \leq n}$ is equal to the order along $\operatorname{det}\left(\partial F_{k} / \partial T_{l}\right)_{k, l \leq r}$, and it is equal to $\mathbf{e}_{r}$; for all $i \leq r-1$, the order along the ideal of $i$-minors of $\left(\partial F_{k} / \partial T_{l}\right)_{k \leq i, l \leq i+1}$

is equal to the order along $\operatorname{det}\left(\partial F_{k} / \partial T_{l}\right)_{k, l \leq i}$, and it is equal to $\mathbf{e}_{i}$. It is clear that $\left(\mathbb{A}^{n}\right)_{p}^{(\mathbf{e})}$ is a locally closed subset of $\left(\mathbb{A}^{n}\right)_{p}$. We put $D_{p}^{(\mathbf{e})}:=D_{p} \cap\left(\mathbb{A}^{n}\right)_{p}^{(\mathbf{e})}$, and let $D_{p, \infty}^{(\mathbf{e})}=D_{p}^{(\mathbf{e})} \cap D_{p, \infty}$. Our goal in this setion is to give equations for $D_{p, \infty}^{(\mathbf{e})}$ inside $\left(\mathbb{A}^{n}\right)_{p}^{(\mathbf{e})}$. Of course, we have $r(p+1)$ equations which cut out $D_{p}$ in $\left(\mathbb{A}^{n}\right)_{p}$, and we will show that in addition to these, we need only $\mathbf{e}_{r}$ extra equations.

We fix first some notation. Let $u \in\left(\mathbb{C}[t] /\left(t^{p+1}\right)\right)^{n}$ be a point in $\left(\mathbb{A}^{n}\right)_{p}^{(\mathbf{e})}$. We denote by $\tilde{u} \in(\mathbb{C}[[t]])^{n}$ the unique lifting of $u$ by polynomials of degree no more than $p$. If we 
denote by $M$ the $r \times r$ matrix which is the classical adjoint of $\left(\partial F_{k} / \partial T_{l}\right)_{k, l \leq r}$, then we have the following

Lemma 2.1. ([DL]) With the above notation, $u \in\left(\mathbb{A}^{n}\right)_{p}^{(\mathbf{e})}$ is in $D_{p, \infty}^{(\mathbf{e})}$ if and only if

$$
\operatorname{ord}(M(\tilde{u}) F(\tilde{u})) \geq p+\mathbf{e}_{r}+1 \text {. }
$$

Proof. It is clear that if $\mathrm{Jac}_{F}$ is the Jacobian matrix of $F$, then

$$
M(\tilde{u}) \cdot \operatorname{Jac}_{F}(\tilde{u})=\left(\operatorname{det}\left(\partial F_{k} / \partial T_{l}\right)_{k, l \leq r}(\tilde{u}) \cdot I_{r}, B(\tilde{u})\right),
$$

for some $r \times(n-r)$ matrix $B$. An easy computation based on Cramer's rule shows that all the entries of $B(\tilde{u})$ have order at least $\mathbf{e}_{r}$.

We have $u \in D_{p, \infty}^{(\mathbf{e})}$ if and only if there is $v \in(t \mathbb{C}[[t]])^{n}$, such that $F\left(\tilde{u}+t^{p} v\right)=0$. Expanding using Taylor's formula gives

$$
F\left(\tilde{u}+t^{p} v\right)=F(\tilde{u})+t^{p} \operatorname{Jac}_{F}(\tilde{u}) v+t^{2 p}(\ldots),
$$

where all the entries in $(\ldots)$ have order at least 2 . As $\operatorname{det}(M(\tilde{u})) \neq 0$, we have $F\left(\tilde{u}+t^{p} v\right)=$ 0 if and only if $M(\tilde{u}) \cdot F\left(\tilde{u}+t^{p} v\right)=0$.

Since $2 p+2 \geq p+\mathbf{e}_{r}+2$, and since $v \equiv 0(\bmod t)$, and $M(\tilde{u}) \operatorname{Jac}_{F}(\tilde{u}) \equiv 0\left(\bmod t^{\mathbf{e}_{r}}\right)$, we deduce that if the above condition is satisfied, then

$$
\operatorname{ord}(M(\tilde{u}) \cdot F(\tilde{u})) \geq p+\mathbf{e}_{r}+1 .
$$

To see that this is also sufficient, note that $M(\tilde{u}) \cdot F\left(\tilde{u}+t^{p} v\right)=0$ can be rewritten as $H_{1}(v)=\ldots=H_{r}(v)=0$, for suitable polynomials $H_{1}, \ldots, H_{r}$ with coefficients in $\mathbb{C}[[t]]$. As $H(0) \equiv 0(\bmod t)$, and the order of an $r$-minor of the Jacobian of $H$ is zero, the existence of $v$ follows from (11) via the Implicit Function Theorem for formal power series.

From now on we will assume that $u \in D_{p}$, so that $\operatorname{ord}(F(\tilde{u})) \geq p+1$. Therefore the condition in (11) amounts to the vanishing of the coefficients of $t^{i}$ in the corresponding $r$ equations, for $p+1 \leq i \leq p+1+\mathbf{e}_{r}$. Therefore we get $r \mathbf{e}_{r}$ equations. Note that while (11) is written in terms of $\tilde{u}$, since the lifting $\tilde{u}$ of $u$ was canonical, the resulting equations lie in the coordinate ring of $\left(\mathbb{A}^{n}\right)_{p}$. In the remaining of this section we will show that, in fact, we may replace the above $r \mathbf{e}_{r}$ equations by just $\mathbf{e}_{r}$ equations. This is the content of the following

Theorem 2.2. $D_{p, \infty}^{(\mathbf{e})}$ can be cut out in $\left(\mathbb{A}^{n}\right)_{p}^{(\mathbf{e})} \cap D_{p}$ by $\mathbf{e}_{r}$ equations.

Proof. We prove the theorem by induction on $r$, the case $r=1$ being an immediate consequence of Lemma 2.1. Let $J$ denote the matrix $\left(\partial F_{k} / \partial T_{l}\right)_{k, l \leq r}$. We denote by $\delta_{i, j}$ the $(r-1)$-minor of $J$, obtained by deleting the $i$ th row and the $j$ th column. Similarly, the $(r-2)$-minor obtained by deleting the $i$ th and the $k$ th rows and the $j$ th and the $l$ th columns will be denoted by $\delta_{i k, j l}$. Here we assume $i \neq k$ and $j \neq l$. We will use the following lemma, whose proof will be given at the end of this setion.

Lemma 2.3. For every $r \times r$ matrix $J$, if $i<k$ and $j<l$, then

$$
\delta_{i, j} \cdot \delta_{k, l}-\delta_{i, l} \cdot \delta_{k, j}=\operatorname{det}(J) \cdot \delta_{i k, j l} .
$$


Suppose that $u \in\left(\mathbb{A}^{n}\right)_{p}^{(\mathbf{e})} \cap D_{p}$. Recall that $M$ is the classical adjoint of $J$, so $m_{i j}=(-1)^{i+j} \delta_{j, i}$. The condition in (11) can be written as a set of $r$ order conditions, the $r$ th one being

$$
\operatorname{ord}\left((-1)^{r} \delta_{r, r}(\tilde{u}) F_{r}(\tilde{u})+\sum_{j=1}^{r-1}(-1)^{j} \delta_{j, r}(\tilde{u}) F_{j}(\tilde{u})\right) \geq p+\mathbf{e}_{r}+1
$$

For every $i \leq r-1$, after multiplying the corresponding expression from (11) by $\delta_{r, r}(\tilde{u})$ (whose order is $\mathbf{e}_{r-1}$ ), we can rewrite the $i$ th condition in (11) as

(3) $\operatorname{ord}\left((-1)^{r} \delta_{r, r}(\tilde{u}) \delta_{r, i}(\tilde{u}) F_{r}(\tilde{u})+\delta_{r, r}(\tilde{u}) \cdot \sum_{j=1}^{r-1}(-1)^{j} \delta_{j, i}(\tilde{u}) F_{j}(\tilde{u})\right) \geq p+\mathbf{e}_{r}+\mathbf{e}_{r-1}+1$.

Granting (2), which we multiply by $\delta_{r, i}(\tilde{u})$ (whose order is at least $\mathbf{e}_{r-1}$ ), we see that the condition in (3) is equivalent with

$$
\operatorname{ord}\left(\sum_{j=1}^{r-1}(-1)^{j} \delta_{j, i}(\tilde{u}) \delta_{r, r}(\tilde{u}) F_{j}(\tilde{u})+\sum_{j=1}^{r-1}(-1)^{j+1} \delta_{r, i}(\tilde{u}) \delta_{j, r}(\tilde{u}) F_{j}(\tilde{u})\right) \geq p+\mathbf{e}_{r}+\mathbf{e}_{r-1}+1 \text {. }
$$

Using Lemma 2.3, this can be further rewritten as

$$
\text { ord }\left(\sum_{j=1}^{r-1}(-1)^{j} \delta_{j r, i r}(\tilde{u}) F_{j}(\tilde{u})\right) \geq p+\mathbf{e}_{r-1}+1 .
$$

Here we used the fact that $\operatorname{ord}(\operatorname{det}(J))=\mathbf{e}_{r}$. If we denote by $J^{\prime}$ the matrix formed by the first $(r-1)$ rows and columns of $J$, and by $M^{\prime}$ the classical adjoint of $J^{\prime}$, and if we put $F^{\prime}=\left(F_{1}, \ldots, F_{r-1}\right)$, then the above conditions (for $\left.i \leq r-1\right)$ can be rewritten as

$$
\operatorname{ord}\left(M^{\prime}(\tilde{u}) \cdot F^{\prime}(\tilde{u})\right) \geq p+\mathbf{e}_{r-1}+1 .
$$

By induction on $r$, this is equivalent with a set of $\mathbf{e}_{r-1}$ equations $G_{1}(u)=\ldots=G_{\mathbf{e}_{r-1}}(u)=$ 0 .

Granting this, we go back to the condition in (2). Since $u \in D_{p}$, we have ord $F(\tilde{u}) \geq$ $p+1$, so that $\operatorname{ord}\left(\delta_{r, r}(\tilde{u}) F_{r}(\tilde{u})\right) \geq p+\mathbf{e}_{r-1}+1$. Moreover, by expanding each $(r-1)$ minor along the last row, we write

$$
\sum_{j=1}^{r-1}(-1)^{j} \delta_{j, r}(\tilde{u}) F_{j}(\tilde{u})=\sum_{i=1}^{r-1}(-1)^{i+r+1} \partial F_{r} / \partial T_{i}(\tilde{u}) \sum_{j=1}^{r-1}(-1)^{j} \delta_{j r, i r}(\tilde{u}) F_{j}(\tilde{u}),
$$

which by (4) has order at least $p+\mathbf{e}_{r-1}+1$. Therefore, in order to ensure (2) we need only $\left(\mathbf{e}_{r}-\mathbf{e}_{r-1}\right)$ more equations $G_{\mathbf{e}_{r-1}+1}(u)=\ldots=G_{\mathbf{e}_{r}}(u)$. This concludes the proof of the theorem.

We give now the prrof of the lemma we have used.

Proof of Lemma 2.3. All the determinants are considered as polynomial functions on the affine space of all matrices. Let $P$ denote the difference of products of minors in the left hand side of the formula in the lemma. To see that $P(A)$ is $\operatorname{divisible}$ by $\operatorname{det}(A)$ 
for every matrix $A$, it is enough to show that if $A$ is such that one column is a linear combination of the other columns, then $P(A)=0$. Let that column be indexed by $m$, so we can write $C_{m}=\sum_{\alpha \neq m} \lambda_{\alpha} C_{\alpha}\left(C_{\alpha}\right.$ denotes the $\alpha$ th column). If $m \neq j, l$, then $\delta_{i, j}(A)=(-1)^{m-j} \lambda_{j} \delta_{i, m}(A), \delta_{k, l}(A)=(-1)^{m-l} \lambda_{l} \delta_{k, m}(A), \delta_{i, l}(A)=(-1)^{m-l} \lambda_{l} \delta_{i, m}(A)$, and $\delta_{k, j}(A)=(-1)^{m-j} \lambda_{j} \delta_{k, m}(A)$. This clearly gives $P(A)=0$.

Suppose now, for example, that $m=j$. We have $\delta_{k, l}(A)=(-1)^{m-l} \lambda_{l} \delta_{k, j}(A)$ and $\delta_{i, l}(A)=(-1)^{m-l} \lambda_{l} \delta_{i, j}(A)$, and this gives $P(A)=0$. The case $m=l$ is similar.

It follows from the above discussion that we can write $P(A)=\operatorname{det}(A) \cdot Q(A)$. Since the degree of $P$ with respect to any of $a_{i \beta}, a_{\beta j}, a_{k \beta}$ and $a_{\beta l}$ is at most one, for all $1 \leq \beta \leq r$, we see that $Q$ depends only on the remaining variables.

Therefore, if $B$ is the matrix obtained from a matrix $A$ by deleting the $i$ th and the $k$ th rows and the $j$ th and the $l$ th columns, then $Q$ depends only on $B$. In order to compute $Q(B)$, we may assume that $a_{i j}=a_{k l}=1$, and that all the other entries on those two rows and columns are zero. In this case, it is clear that $\delta_{i, j}(A)=(-1)^{k+l} \delta_{i k, j l}(A), \delta_{k, l}(A)=$ $(-1)^{i+j} \delta_{i k, j l}(A)$, while $\delta_{i, l}(A)=0=\delta_{k, j}(A)$. Since $\operatorname{det}(A)=(-1)^{k+l+i+j} \delta_{i k, j l}(A)$, we see that $Q(A)=\delta_{i k, j l}(A)$, as required.

Remark 2.4. Note that the above description of the equations does not assume that $D$ is locally complete intersection, but only that the number of equations is not greater than the dimension of the affine space.

\section{Log Discrepancies ANd Inversion of AdJunction}

We start by recalling the definition of the version of minimal log discrepancies from EMY. Consider a pair $(X, Y)$, where $X$ is a $\mathbb{Q}$-Gorenstein normal variety, and where $Y$ stands for a formal combination $\sum_{i=1}^{k} q_{i} \cdot Y_{i}$, where $q_{i} \in \mathbb{R}_{+}$and $Y_{i} \subset X$ are proper closed subschemes. A divisor $E$ over $X$ is a prime divisor on some smooth variety $X^{\prime}$, such that we have a proper, birational morphism $f: X^{\prime} \longrightarrow X$. In fact, we identify two such divisors if they give the same valuation of the function field of $X$. In particular, we may assume that the inverse image of each $Y_{i}$ on $X^{\prime}$ is an effective Cartier divisor. The center of $E$ on $X$ is $c_{X}(E):=f(E)$.

The log discrepancy $a(E ; X, Y)$ is defined such that $a(E ; X, Y)-1$ is the coefficient of $E$ in $K_{X^{\prime} / X}-f^{-1}(Y)$. Here $f^{-1}(Y)=\sum_{i} q_{i} f^{-1}\left(Y_{i}\right)$, where $f^{-1}\left(Y_{i}\right)$ is the scheme theoretic inverse image of $Y_{i}$. Recall the convention that $K_{X^{\prime} / X}$ is a divisor supported on the exceptional locus of $f$.

If $W \subseteq X$ is a closed subset, then the minimal log discrepancy of $(X, Y)$ on $W$ is defined by

$$
\operatorname{mld}(W ; X, Y):=\min _{c_{X}(E) \subseteq W} a(E ; X, Y) .
$$

For more on these invariants, we refer to [EMY], Section 1, or to [Am].

We mention that the pair $(X, Y)$ is called $\log$ canonical if $\operatorname{mld}(X ; X, Y) \geq 0$. This a requirement for having interesting such invariants: if $\operatorname{dim} X \geq 2$, and if $(X, Y)$ is not $\log$ canonical, then $\operatorname{mld}(X ; X, Y)=-\infty$. Note also that if $\operatorname{mld}(W ; X, Y) \geq 0$, then the pair $(X, Y)$ is $\log$ canonical in some neighbourhood of $W$. 
We need some preparations in order to state the characterization of minimal log discrepancies from [EMY]. We assume from now on that $X$ is a normal, locally complete intersection variety, as this is the setting we have in Theorem 1.1. Let $d=\operatorname{dim} X$, and let $Z \subset X$ be the Jacobian subscheme of $X$, i.e., the subscheme defined by the $d$ th Fitting ideal of $\Omega_{X}^{1}$. Note that the support of $Z$ is the singular locus $X_{\text {sing }}$ of $X$.

If $Y \subset X$ is a closed subscheme, then we have a function $F_{Y}: X_{\infty} \longrightarrow \mathbb{N} \cup\{\infty\}$, such that $F_{Y}(\gamma)$ is the order of vanishing of $\gamma$ along the ideal of $Y$. Recall that we have canonical projections $\psi_{m}^{X}: X_{\infty} \longrightarrow X_{m}$, so that $F_{Y}^{-1}(\geq m)=\psi_{m-1}\left(Y_{m-1}\right)$, for all $m \geq 1$.

It is a theorem of Denef and Loeser from DL that if $m \geq e$, then the induced projections

$$
\psi_{m+1}^{X}\left(F_{Z}^{-1}(e)\right) \longrightarrow \psi_{m}^{X}\left(F_{Z}^{-1}(e)\right)
$$

are locally trivial, with fiber $\mathbb{A}^{d}$. Suppose now that we have proper closed subschemes $Y_{1}, \ldots, Y_{k} \subset X$, and suppose that $A=\bigcap_{i} F_{Y_{i}}^{-1}\left(\geq m_{i}\right)$, for some $m_{i} \in \mathbb{N}$. The codimension of $A$ in $X_{\infty}$ is defined by

$$
\operatorname{codim}\left(A, X_{\infty}\right):=\min _{e, m}\left\{(m+1) d-\operatorname{dim} \psi_{m}^{X}\left(A \cap F_{Z}^{-1}(e)\right),\right.
$$

where the minimum is over all $e \in \mathbb{N}$ and $m \geq \max \left\{e, m_{1}, \ldots, m_{k}\right\}$ (the above result of Denef and Loeser implies that the expression in (15) is independent of which $m$ we choose, as above). We use the convention $\operatorname{dim}(\emptyset)=-\infty$.

Theorem 3.1. ([EMY] $)$ Let $(X, Y)$ be a pair, with $X$ a normal, locally complete intersection variety, and $Y=\sum_{i=1}^{k} q_{i} \cdot Y_{i}$, where $q_{i} \in \mathbb{R}_{+}$, and where $Y_{i} \subset X$ are proper closed subschemes. Fix a proper closed subscheme $W \subset X$ and let $\tau \in \mathbb{R}_{+}$. If $Z \subset X$ is the Jacobian subscheme, then $\operatorname{mld}(W ; X, Y) \geq \tau$ if and only if

$$
\operatorname{codim}\left(F_{W}^{-1}(\geq 1) \cap F_{Z}^{-1}(\geq e) \cap \bigcap_{i=1}^{k} F_{Y_{i}}^{-1}\left(\geq m_{i}\right), X_{\infty}\right) \geq e+\sum_{i=1}^{k} q_{i} m_{i}+\tau
$$

for every $e \in \mathbb{N},\left(m_{i}\right)_{i} \in \mathbb{N}^{k}$.

We combine now the above theorem with our result from the previous section to give a proof of the case of the Inversion of Adjunction Conjecture which was stated in Section 1.

Proof of Theorem 1.1. The inequality

$$
\operatorname{mld}(W ; X, D+Y) \leq \operatorname{mld}\left(W ; D,\left.Y\right|_{D}\right)
$$

is well-known in general and follows by adjunction (see, for example, the proof of Proposition 7.3.2 in $\mathrm{K}+$, or the proof of Theorem 1.6 in [EMY]). We give the proof of the reverse inequality.

Working locally, we may assume that $X$ is a subvariety of codimension $r-1$ of an open subset $U$ of an affine space $\mathbb{A}^{n}$. Moreover, we may assume that $X=H_{1} \cap \ldots H_{r-1}$, 
and $D=X \cap H_{r}$, where $H_{i}$ is defined in $U$ by $F_{i} \in \mathbb{C}\left[T_{1}, \ldots, T_{n}\right]$. An inductive application of (6) gives

$$
\operatorname{mld}\left(W ; U, Y+\sum_{i=1}^{r} H_{i}\right) \leq \operatorname{mld}(W ; X, D+Y)
$$

Therefore it is enough to prove $\operatorname{mld}\left(W ; D,\left.Y\right|_{D}\right) \leq \operatorname{mld}\left(W ; U, Y+\sum_{i=1}^{r} H_{i}\right)$.

Consider $\tau \in \mathbb{R}_{+}$, and suppose that $\operatorname{mld}\left(W ; D,\left.Y\right|_{D}\right) \geq \tau$. We assume that $\operatorname{mld}(W ; U, Y+$ $\left.\sum_{i=1}^{r} H_{i}\right)<\tau$, and we will derive a contradiction. We first apply Theorem 3.1 on the smooth variety $U$, to deduce that we can find $m_{1}, \ldots, m_{k} \in \mathbb{N}$, and $m_{1}^{\prime}, \ldots, m_{r}^{\prime} \in \mathbb{N}$ such that

$$
\operatorname{codim}\left(F_{W}^{-1}(\geq 1) \cap \bigcap_{i=1}^{k} F_{Y_{i}}^{-1}\left(\geq m_{i}\right) \cap \bigcap_{j=1}^{r} F_{H_{j}}^{-1}\left(\geq m_{j}^{\prime}\right), U_{\infty}\right)<\sum_{i=1}^{k} m_{i} q_{i}+\sum_{j=1}^{r} m_{j}^{\prime}+\tau .
$$

Let us choose $m \geq m_{i}, m_{j}^{\prime}$, for all $i$ and $j$, and consider the projection to $U_{m}$ of the above subset of $U_{\infty}$. We may choose an irreducible component $V$ of this projection, such that $\operatorname{dim} V>(m+1) n-\sum_{i} q_{i} m_{i}-\sum_{j} m_{j}^{\prime}-\tau$.

As in Lemma 3.2 from EMY, one can show that $V \cap \psi_{m}^{D}\left(D_{\infty}\right) \neq \emptyset$. Moreover, given $v \in V \cap\left(\psi_{m}^{D}\right)\left(D_{\infty}\right)$, there is $\tilde{v} \in\left(\psi_{m}^{D}\right)^{-1}(v) \backslash Z_{\infty}$. Here $Z \subset D$ is the Jacobian subscheme of $D$, which is cut out on $D$ by the $r$-minors of the matrix $\left(\partial F_{i} / \partial T_{j}\right)_{i \leq r, j \leq n}$. If $f=F_{Z}(\tilde{v})$, then we may assume that $f \leq m$. Indeed, otherwise, we may just replace $m$ by some $m^{\prime} \geq f, V$ by its inverse image in $X_{m^{\prime}}$, and $v$ by $\psi_{m^{\prime}}^{D}(\tilde{v})$.

Choose now $\mathbf{e}_{r}$ to be the smallest order of vanishing along $Z$ of an element in $V \cap \operatorname{Im}\left(\psi_{m}^{D}\right)$. We see that $\mathbf{e}_{r} \leq f \leq m$. After renumbering the variables, we may assume that some element in $\left(\psi_{m}^{D}\right)^{-1}(V)$ vanishes along $\operatorname{det}\left(\partial F_{i} / \partial X_{j}\right)_{i, j \leq r}$ with order $\mathbf{e}_{r}$. For these elements, we choose $\mathbf{e}_{r-1} \leq \mathbf{e}_{r}$ to be the smallest order of vanishing along the ideal generated by the $(r-1)$-minors of $\left(\partial F_{i} / \partial X_{j}\right)_{i \leq r-1, j \leq r}$. Moreover, after renumbering the variables we may assume that this minimum is achieved by an element as above, along $\operatorname{det}\left(\partial F_{i} / \partial X_{j}\right)_{i, j \leq r-1}$. We proceed in this way to obtain the sequence $\mathbf{e}_{1} \leq \mathbf{e}_{2} \leq \ldots \leq \mathbf{e}_{r}$. We fix also $\tilde{v}^{\prime} \in\left(\psi_{m}^{D}\right)^{-1}\left(V \cap\left(\mathbb{A}^{n}\right)_{m}^{(\mathbf{e})}\right)$, where we use the notation introduced in the previous section.

By Greenberg's theorem (see $\mathrm{Gr}$ ), there is $p \geq m$ such that every element in $D_{m}$ which can be lifted to $D_{p}$ can be lifted to $D_{\infty}$. If $\phi: D_{p} \longrightarrow D_{m}$ is the canonical projection, then let $V^{\prime}$ be an irreducible component of $\phi^{-1}\left(V \cap D_{m}\right)$ which contains $\psi_{p}^{D}\left(\tilde{v}^{\prime}\right)$. Note that $\phi^{-1}\left(V \cap D_{m}\right)$ is cut out in $V \times \mathbb{A}^{(p-m) n}$ by $\sum_{j=1}^{r}\left(p-m_{j}^{\prime}+1\right)$ equations, we see that

$$
\operatorname{dim} V^{\prime}>(p+1)(n-r)-\sum_{i=1}^{k} q_{i} m_{i}-\tau \text {. }
$$

It follows from our choice of e and $p$ that $V_{0}^{\prime}:=V_{0} \cap\left(\mathbb{A}^{n}\right)_{p}^{(\mathbf{e})}$ is open in $V^{\prime}$. Moreover, Theorem 2.2 shows that $V_{0}^{\prime \prime}:=V_{0}^{\prime} \cap \operatorname{Im}\left(\psi_{p}^{D}\right)$ is cut out in $V_{0}^{\prime}$ by $\mathbf{e}_{r}$ equations. Since $V_{0}^{\prime \prime}$ is nonempty, by construction, we see that

$$
\operatorname{codim}\left(\left(\psi_{p}^{D}\right)^{-1}\left(V_{0}^{\prime \prime}\right), D_{\infty}\right)<\mathbf{e}_{r}+\sum_{i=1}^{k} q_{i} m_{i}+\tau
$$


while $\left(\psi_{p}^{D}\right)^{-1}\left(V_{0}^{\prime \prime}\right) \subseteq F_{W}^{-1}(\geq 1) \cap F_{Z}^{-1}\left(\geq \mathbf{e}_{r}\right) \cap \bigcap_{i} F_{Y_{i}}^{-1}\left(\geq m_{i}\right)$. This contradicts $\operatorname{mld}\left(W ; D,\left.Y\right|_{D}\right) \geq$ $\tau$, by Theorem [3.1] and completes the proof.

Corollary 3.2. Let $V$ be a smooth variety, $X \subset V$ a normal, locally complete intersection subvariety of codimension $r$, and $Y=\sum_{i} q_{i} \cdot Y_{i}$, where $q_{i} \in \mathbb{R}_{+}$and where $Y_{i} \subset X$ are proper closed subschemes. If $W \subset X$ is a proper closed subset, then $\operatorname{mld}(W ; X, Y)=$ $\operatorname{mld}(W ; V, Y+r \cdot X)$.

Proof. Working locally, we may assume that $X=H_{1} \cap \ldots \cap H_{r}$, where the $H_{i}$ 's are effective divisors on $V$. Note that $H_{1} \cap \ldots \cap H_{i}$ is normal around $X$, for every $i$. It is enough to show that we have the inequalities

$$
\operatorname{mld}\left(W ; V, Y+\sum_{i=1}^{r} H_{i}\right) \leq \operatorname{mld}(W ; V, Y+r \cdot X) \leq \operatorname{mld}(W ; X, Y),
$$

as the extremal terms are equal by an inductive application of Theorem 1.1 The first inequality follows immediately from the definition of minimal log discrepancies.

For the second one, we proceed as follows. Let $\pi: \widetilde{V} \longrightarrow V$ be the blowing-up of $V$ along $X$, with exceptional divisor $E$. If $\pi^{\prime}: E \longrightarrow X$ is the restriction of $\pi$, then $\pi^{\prime}$ is a projective bundle with fiber $\mathbb{P}^{r-1}$, so that $\operatorname{mld}(W ; X, Y)=\operatorname{mld}\left(\pi^{-1}(W) ; E, \pi^{-1}(Y)\right)$. Moreover, $E$ is reduced and irreducible. It is also a locally complete intersetion, hence so is $\widetilde{V}$. By computing $K_{\widetilde{V} / V}$ at the general point of $E$, we see that $K_{\widetilde{V} / V}=(r-1) E$.

From the formula describing the behaviour of minimal log discrepancies under a proper, birational morphism (see [EMY], Proposition 1.3 (iv)), we get

$$
\begin{aligned}
& \operatorname{mld}(W ; V, Y+r \cdot X)=\operatorname{mld}\left(\pi^{-1}(W) ; \tilde{V}, \pi^{-1}(Y)+r E-K_{\widetilde{V} / V}\right) \\
& =\operatorname{mld}\left(\pi^{-1}(W) ; \widetilde{V}, \pi^{-1}(Y)+E\right) \leq \operatorname{mld}\left(\pi^{-1}(W) ; E, \pi^{-1}(Y)\right),
\end{aligned}
$$

where the inequality follows from (6). Therefore $\operatorname{mld}(W ; V, Y+r \cdot X) \leq \operatorname{mld}(W ; X, Y)$, so we are done.

Together with the corresponding result from [EMY], this immediately gives the semicontinuity of minimal log discrepancies on locally complete intersetion varieties.

Proof of Theorem 1.2. Working locally, we may assume that $X$ is a codimension $r$ subvariety of a smooth variety $V$. It follows from the above corollary that for every $x \in X$, we have

$$
\operatorname{mld}(x ; X, Y)=\operatorname{mld}(x ; V, Y+r \cdot X),
$$

so it is enough to use the corresponding semicontinuity assertion on smooth varieties. This is Theorem 0.3 in EMY.

We give now the proof of the characterization of certain classes of singularities for locally complete intersection varieties. 
Proof of Theorem 1.3. Recall the following characterization of log canonical, canonical, and terminal singularities in terms of minimal log discrepancies. A normal, $\mathbb{Q}$-Gorenstein variety $X$ has log canonical (canonical, terminal) singularities if and only if we have $\operatorname{mld}\left(X_{\text {sing }} ; X, \emptyset\right) \geq 0$ (respectively, $\geq 1,>1$ ). Note also that if $X$ is Gorenstein, as in our case, then the above minimal log discrepancy is an integer.

We consider first the characterization of $\log$ canonical singularities. Let $\pi_{m}: X_{m} \longrightarrow$ $X$ be the canonical projection. $X_{m}$ is equidimensional if and only if $\operatorname{dim} \pi_{m}^{-1}\left(X_{\operatorname{sing}}\right) \leq$ $(m+1) \operatorname{dim} X$. Moreover, if this is the case, then $X_{m}$ is a locally complete intersection and $\operatorname{dim} X_{m}=(m+1) \operatorname{dim} X$ (see $\mathrm{Mu} 2$ ). Working locally, we may assume that $X$ is a codimension $r$ subvariety of a smooth variety $V$. It follows from Theorem 3.1 that $X_{m}$ is equidimensional for every $m$ if and only if $\operatorname{mld}\left(X_{\text {sing }} ; V, r \cdot X\right) \geq 0$. By Corollary 3.2 this is equivalent with $\operatorname{mld}\left(X_{\text {sing }} ; X, \emptyset\right) \geq 0$, i.e., with $X$ having $\log$ canonical singularities. This completes this case.

Similarly, $X_{m}$ is irreducible if and only if $\operatorname{dim} \pi_{m}^{-1}\left(X_{\text {sing }}\right) \leq(m+1) \operatorname{dim} X-1$ (see $\mathrm{Mu} 2$ ). The characterization of canonical singularities follows as above. From now on, we may assume that $X$ has canonical singularities. In particular, $X_{m}$ is a locally complete intersection for every $m$. Serre's criterion implies that $X_{m}$ is normal if and only if $\operatorname{dim}\left(X_{m}\right)_{\operatorname{sing}} \leq(m+1) \operatorname{dim} X-2$. One can easily show that because $X_{m}$ is equidimensional, we have $\left(X_{m}\right)_{\text {sing }}=\pi_{m}^{-1}\left(X_{\text {sing }}\right)$ (see the proof of Theorem 3.3 in [EMY] $)$. Therefore $X_{m}$ is normal for every $m$ if and only if $\operatorname{mld}\left(X_{\text {sing }} ; V, r \cdot X\right) \geq 2$, and we conclude as above, applying Corollary 3.2 .

Remark 3.3. It is clear that if $X$ is a variety such that $X_{1}$ is irreducible, then $\operatorname{dim} X_{\text {sing }} \leq$ $\operatorname{dim} X-2$. If $X$ is locally a complete intersection, then $X$ has to be normal. It follws that in the above characterization for canonical or terminal singularities, we do not need to assume that $X$ is normal.

Acknowledgements. We are grateful to Florin Ambro, Vyacheslav V. Shokurov, and Takehiko Yasuda for useful discussions. The first author was partially supported by NSF grant DMS 0200278. The second author served as a Clay Mathematics Institute LongTerm Prize Fellow while this research has been done.

\section{REFERENCES}

[Am] F. Ambro, On minimal log discrepancies, Math. Res. Letters 6 (1999), 573-580.

[DL] J. Denef and F. Loeser, Germs of arcs on singular varieties and motivic integration, Invent. Math. 135 (1999), 201-232.

[EMY] L. Ein, M. Mustaţă and T. Yasuda, Log discrepancies, jet schemes and Inversion of Adjunction, math.AG/0209392

[Gr] M. Greenberg, Rational points in henselian discrete valuation rings, Publ. Math. I.H.E.S. 31 (1966), 59-64.

[K+] J. Kollár (with 14 coauthors), Flips and abundance for algebraic threefolds, Astérisque 211, 1992.

[Mu2] M. Mustaţă, Jet schemes of locally complete intersection canonical singularities, with an appendix by D. Eisenbud and E. Frenkel, Invent. Math. 145 (2001), 397-424. 
Department of Mathematics, Statistics and Computer Science, University of Illinois at Chicago, 851 Morgan St., M/C. 249, Chicago, IL 60607-7045, USA

E-mail address: ein@math.uic.edu

Department of Mathematics, Harvard University, One Oxford Street, Cambridge, MA 02138, USA

E-mail address: mirceamustata@yahoo.com 